\title{
ON THE PATHOLOGY
}

\section{AND \\ TREATMENT OF CHOLERA.}

BY

GEORGE JOHNSON, M.D.,

PHYSICIAN TO KING'S COLLEGK HOSPITAI.

Received Feb. 26th.--Read April 23rd, 1867.

I HAvE ventured to bring this subject before the Royal Medical and Chirurgical Society in the hope and expectation that a full discussion will tend to establish something like an agreement amongst those who are competent to form an opinion, as to the pathological nature of cholera, and the priuciples on which its treatment should be conducted. I will proceed at once to state the main conclusions at which, after a careful study of the disease, $I$ have myself arrived, together with the reasons which have led me to these conclusions. Upon these I invite comment and criticism.

The phenomena of cholera result from the action of a morbid poison.-There are very few pathologists who dispute this proposition. That the disease is portable and communicable, mainly, perhaps, by means of the discharges from the alimentary canal, is generally admitted. 
The very general use of disinfectants during the recent epidemic implies a wide-spread belief that there is a material poison to be destroyed.

The doctrine of the communicability of cholera from the sick to the healthy is by no means inconsistent with the fact that cases sometimes occur where it is impossible to trace the disease to any source of infection. A peculiar but unknown state of the atmosphere may convey the disease without the agency of human intercourse.

The poison is often swallowed, and enters the system through the alimentary canal.-This appears to be proved by the undoubted fact that impure water is, in a very large proportion of cases, the vehicle of the poison.

The poison is sometimes taken in with the air through the lungs.-This is rendered nearly certain by such cases as that referred to by Dr. Wm. Budd, ${ }^{1}$ in which the spread of cholera through a large workhouse was clearly traced to the gaseous exhalations from infected privies. Numerous inmates who, from various causes, had not access to the infected privies entirely escaped the disease, which created frightful havoc amongst others-the food and the water being the same for the whole establishment.

Another instance in which the poison must have been conveyed through the air is afforded by the case of the two pilots who took the disease in consequence of having their open boat towed at a considerable distance astern of the steamship England, on board of which cholera was raging. ${ }^{2}$ The pilots were never on board the infected vessel, yet both had cholera : one man died, and both took the disease home to their families near Halifax, where no case of cholera had occurred for years before.

In whatever way the morbid poison is received into the system, whether with the air through the lungs or with the water or food through the stomach, it enters the circulation before it gives rise to its characteristic effects.-This propo-

1 'Association Journal,' 1854, p. 974.

2 'Statistical, Sanitary, and Medical Reports,' Army Medical Department, 1866; also 'British Medical Journal,' November, 1866, p. 505. 
sition is merely the statement of a general law which is applicable to every known poison, whether mineral, vegetable, or animal, whose action is not obviously that of a merely local irritant. Even such comparatively insoluble substances as calomel and arsenic very quickly find their way into the blood and the tissues, while the more soluble and liquid poisons enter the circulation and pervade the whole system with marvellous rapidity. To deny, as some pathologists have done, that the cholera-poison enters the blood is to assert that, in the case of this particular poison, a general law of physiology is suspended.

The fact that the poison, though more commonly swallowed, is sometimes inhaled, yet that however introduced into the system it gives rise to essentially the same symptoms, would alone render it probable that the blood is the receptacle of the poison, and the vehicle by which its influence is transmitted to the various tissues and organs.

Symptoms of invasion.-After the introduction of the poison into the system there is a period, varying from a few hours to two or three days, during which there may be no symptoms of disease-the poison is latent; but in a large propurtion of cases careful observation reveals the existence of symptoms of general constitutional disturbance, probably dependent on blood contamination. In a military report on cholera in Malta in $1865,{ }^{1}$ it is stated that out of fortytwo cases which were carefully noted, these symptoms existed clearly and unmistakably in twenty-seven. The symptoms were a dull and listless appearance of the countenance and eyes; a dark rim round the orbits; a loss of all activity ; want of appetite ; prostration ; vertigo ; cramps; increased perspiration and coldness of the surface on the least-exertion; thirst and general malaise. After these symptoms had continued for a period varying in different cases from a few hours to two or three days, diarrhœa ensued, and the disease was then said to be present. These symptoms, which mark the invasion of cholera, have been

1 'Statistical, Sanitary, and Medical Reports,' Army Medical Department, 1866 ; also ' British Medical Journal,' October, 1866, p. 409. 
described by several of the Indian practitioners. Thus, Twining says," "Prior to the more distinct and alarming attack, there are sometimes for a few hours, and in some cases for two or three days, symptoms of indisposition, evident not only to the patient himself but to his friends. When cholera is raging severely the disease is often ushered in by diarrhœa; at other times it begins with catarrh, nausea, and oppression at the scrobiculus cordis, which are not in an early stage to be distinguished from the slight indisposition which often precedes fever. The approach of cholera in this manner makes the patient suppose he is bilious or feverish, and, if recourse be had to some of the medicines commonly used in slight ailments of that sort, the disease is said to be caused by the dose of medicine taken, when in fact it had been insidiously making progress for some hours."

The symptoms here referred to are probably due to the influence of the morbid poison and its products in the blood upon the nervous system and other structures of the body.

Following more or less rapidly upon the symptoms of invasion, or without being preceded by such symptoms, there occur in most cases of the fully developed disease the diarrhœa stage, with vomiting and purging; the stage of collapse, and the stage of reaction, either with or without consecutive fever of variable severity and duration. To describe these stages is, on the present occasion, wholly unnecessary. I may assume that every one who hears this communication is thoroughly familiar with the phenomena of the disease. The main question which I desire to discuss with this learned Society is this, What is the Pathology of cholera collapse? Then, as subsidiary to this question, we have to inquire in what respect the collapse of cholera resembles, and in what particulars it differs from other wellknown forms of collapse.

The chief forms of collapse-not choleraic - are these :-

1 ' Clinical Illustrations of the more important Diseases of Bengal,' 2nd edition, 1835 , p. 9. 
1st. Collapse from loss of blood or of blood constituents, as from excessive purging.

2nd. Collapse from nervous shock, as from severe mechanical injuries, or from the pain of perforating ulcer of the stomach or bowel.

3rd. Collapse resulting from the operation of certain poisons - as tobacco, digitalis, antimony.

In what respects does the collapse of cholera differ from each of these varieties of collapse?

It differs from the results of loss of blood or of blood constituents, chiefly in these particulars.

1st. The abseuce of a direct and constant relationship between the loss of blood constituents and the degree of collapse. Often there is an inverse relation between collapse and loss of liquid by vomiting and purging. This is a fact admitted by every authority on the subject.

2nd. The discharges invariably continue more or less while the symptoms of collapse are passing off. Death may occur without discharges from the alimentary canal; recovery never.

3rd. In the collapse of cholera, there is blueness, more or less derangement of respiration, often urgent dyspncea, and extreme feebleness or total absence of the pulse without syncope. In the state of collapse from profuse purging there is pallor without dyspnca, and a tendency to faint when the head is raised.

Dr. Macpherson in his work ('Cholera in its Home') quotes from Dr. Pereira a case of poisoning by two and a half drachms of croton oil, as an example of collapse with blueness from excessive purging. In that case the symptoms of collapse began within three-quarters of an hour after the dose was swallowed, and were fully developed within an hour; yet neither vomiting nor purging occurred until half an hour later. It is probable that the blueness and other symptoms of collapse were mainly due to the fact that the oil, having entered the blood, impeded the circulation through the lungs. And this is rendered still more probable by another case recorded by Pereira, in which a 
man who was poisoned by inhaling the dust of croton seeds, was " in a state of collapse," although he had no action of the bowels until several doses of castor oil had been given to him.

4th. "Again, the way in which remedies tell upon the two contrasted conditions is totally and instructively unlike. The coldness and faintness of exhaustion from loss of blood constituents are relieved at once by a glass of wine or brandy : the pulse instantly acknowledges the virtue of the stimulus. But alcoholic stimulants do not warm or invigorate, even for a moment, the patient in choleraic collapse; rather they seem to make matters worse. On the other hand, venesection has often brought marvellous relief under collapse; while to draw blood from a patient who is fainting from exhaustion would probably ensure his death, and would certainly aggravate his danger."

5th. Lastly, recovery from the collapse of cholera is often as rapid as the onset of the symptoms. Whereas, recovery from the exhausting influence of a profuse drain from the blood is always slow.

The collapse from nervous shock and that which results from the depressing influence of such poisons as tobacco, digitalis, and antimony, resemble in their main features that which follows upon an exhausting drain of blood-constituents. There is coldness and pallor of the surface, and extreme feebleness of the pulse, with a tendency to syncope. These symptoms are always more or less decidedly relieved by alcoholic stimulants, and would certainly be aggravated by loss of blood.

Now there is one condition which is common to all these forms of collapse, and that is a defective circulation of blood. Moving blood is the life of the body and of every organ and tissue of which it is made up. A complete arrest of the circulation involves instant death; a great impediment to the circulation brings on a state of collapse which is near to death, and which in extreme cases ends in death. This defective circulation occurs in various ways.

1st. In cases of collapse from hæmorrhage or from exces- 
sive purging there is an absolute deficiency of blood-constituents in the vessels.

2. In cases of nervous shock there has been no loss of blood-constituents, but the heart's action is so enfeebled that the supply of moving blood is greatly diminished.

3. In cases of poisoning by tobacco, digitalis, or antimony, the apparent exhaustion is mainly due to the paralysing influence of the poison upon the heart, and the consequent defective circulation.

4. Lastly, in the collapse of cholera the main and essential cause of the defective circulation is not the loss of blood-constituents, as in cases of hæmorrhage or excessive purging - not an enfeebled condition of the heart induced by the paralysing influence of nervous shock or the action of a depressing poison, but a mechanical arrest of the blood in its passage through the minute vessels of the lungs.

The proofs of this proposition are so numerous, so varied, and so complete, as to render it in my judgment indisputably true. They are derived from a consideration of-

1st. 'The anatomical characters of the state of collapse.

2nd. The harmony of these anatomical characters with the symptoms observed during life, and with the effect of certain modes of treatment.

3rd. The analogous effect of certain accidents occurring in the human subject, and of certain experiments on animals.

I will now, as briefly as is consistent with my statements being made intelligible, refer to the chief facts and arguments upon which I rely to establish the truth of this proposition.

The chief anatomical appearances observed after death during collapse, which prove an impeded flow of blood through the lungs are these: The right side of the heart, the pulmonary artery and the large systemic veins are usually full and even distended with blood, which escapes in large amount when a vessel is punctured or when the vessels at the root of the lung are cut across. The coronary veins are much distended, and usually there are specks of 
ecchymosis beneath the pericardium, the result apparently of great venous turgescence during life. The left side of the heart is comparatively empty. The minute tissue of the lung contains much less than the usual amount of blood and air. The comparative emptiness of the minute pulmonary vessels is shown by the pale look and the dry feel of the lung, but better still by its diminished weight. Dr. Parkes found the average weight of both lungs in twentytwo European soldiers who had died in collapse to be twentysix ounces and six drachms ; and he remarks " if the average weight of both lungs in adult males be considered to be forty-six ounces according to Dr. Clendinning, or fortythree ounces according to Dr. Reid, then the diminution in the weight of both lungs in cholera is shown to be either seventeen or twenty ounces."

Dr. Horace Jeaffreson, in a recently published paper,' gives the weight of the lungs in four cases of collapse. In one man the two lungs weighed nineteen and a half ounces. In three women the average weight of both lungs was twenty. three ounces.

I had few opportunities of making post-mortem examinations during the recent epidemic. In three cases of collapse the lungs were weighed : in one man they weighed seventeen ounces; in another man, seventeen and a half ounces; and in a boy aged fourteen they weighed fifteen ounces.

The diminished weight of the lungs is a pretty accurate measure of the degree of anæmia of their minute vessels. The degree of collapse of the lung, too, will be greater in proportion to the emptiness of the capillaries. After death it usually happens that more or less blood gravitates into the lower parts of the lungs, and hence it is often stated that the lungs are "congested," but a local post-mortem congestion is no index of the state of the pulmonary vessels during life.

In order to ascertain the exact condition of the lungs, heart, and great vessels during life, it is obvious that the

$$
1 \text { 'Edinburgh Medical Journal,' December, } 1866 .
$$


examination should be made as soon as possible after death. The importance of this is well illustrated by the following observation of Harvey." He says, "I have several times opened the breast and pericardium of a man within two hours after his execution by hanging, and before the colour had totally left the face, and, in presence of many wituesses, have demonstrated the right auricle of the heart and the lungs distended with blood; the auricle in particular of the size of a large man's fist, and so full of blood that it looked as if it would burst. This great distension, however, had disappeared next day, the blood having made its escape through various channels."

It is probable that, apart from the influence of gravitation, the tendency of the blood to shift its position after death will be greater in proportion to the tension of the right cavities and the pulmonary artery. So that, when the vital contractility of the small arteries has ceased, the blood is driven onwards by the resiliency of the distended pulmonary artery. The blood then flows through the pulmonary capillaries into the veins which had before been comparatively empty, and thus the equilibrium of pressure is restored by a post-mortem movement of the blood.

It is manifest that the cause and the degree of the impediment which checks the flow of blood through the lungs during the collapse of cholera can be truly estimated only by a comparison of the whole of the symptoms during life, with the whole series of post-mortem phenomena; due allowance being made for such changes in the distribution of the blood in the heart and lungs as are the result of purely physical forces acting after death.

When a body is examined several hours after death it is probable enough that much of the blood, which at the moment of death was accumulated in the right cavities and in the pulmonary artery, will have been pressed onwards through the capillaries into the veins, and even into the left side of the heart. But it is physically impossible that the

1 'Second Disquisition on the Circulation of the Blood,' Sydenham Society's Translation, p. 127. 
blood in any quantity can ever take the opposite course after death, namely, from the capillaries into the pulmonary artery. Anæmia of the minute tissue of the lung after death affords positive evidence of anæmia during life, but engorgement of the lungs may be and often is the result of purely physical changes occurring after death.

When death occurs during the stage of reaction and consecutive fever, the lungs are often, though not necessarily or constantly much engorged, and their weight is increased in a corresponding degree. Dr. Jeaffreson gives the weight of the lungs in three cases fatal after reaction. In a man the two lungs weighed $55 \frac{1}{4}$ ozs. In one woman they weighed $38 \frac{1}{2}$ ozs., and in another woman 53 ozs. The capillary engorgement of the lungs sometimes comes on immediately after the commencement of reaction. There is a rise of temperature, an imperfect rally, quickly followed by hurried breathing, drowsiness, and death. The lungs are found much gorged, and these cases are sometimes reported as cases of death in collapse, with congested lungs; but that reaction had commenced is conclusively shown by the presence of bile in the small intestines.

The opposite states of the lung during collapse and reaction may be compared-the one with the pale and shrunker appearance of the hands when the vessels are contracted by cold, and the other with the florid and turgid condition of the same parts when, with returning warmth, the vessels are relaxed, and there is an unusual afflux of blood.

The anatomical condition of the lungs, the heart, and the great vessels, is in strict harmony with the symptoms observed during life. During the stage of collapse the systemic arteries are so empty of blood that the radial pulse is often not to be felt, while the venous system is so full that the whole surface of the body is more or less livid.

The lividity is usually greatest when collapse has come on suddenly with but little loss of fluid. In such cases the systemic veins are distended to their minutest extremities : on the other hand, when profuse discharges precede or 
accompany collapse, the fluid contents of the vessels being much diminished, there is no fulness of the superficial veins, and therefore little or no blueness.

What then is the difference between the collapse of cholera and ordinary asphyxia? In ordinary asphyxia the systemic arteries are preternaturally distended, because imperfect aërated blood is impeded in its transit through the minute systemic vessels. Whereas, in the collapse of cholera the systemic arteries, receiving a scanty stream of duly oxygenised blood, readily transmit it, and are therefore preternaturally empty. The impeded transit of unaërated black blood through the systemic vessels, is conclusively proved by Dr. John Reid's experiments on asphyxia.

In ordinary asphyxia the patient is more or less drowsy in consequence of the circulating blood containing an excess of carbonic acid. In cholera collapse the arterial blood does not contain an excess of carbonic acid, and the patient is not sleepy ; but his brain is simply inactive in consequence of the scanty supply of arterial blood which it receives.

In ordinary asphyxia the lividity of the surface is due partly to distension of the superficial veins, partly to the circulation of black blood through the arteries. In cholera collapse it is due to the former cause alone; when that is not present lividity is absent, and the lips of a patient in pulseless collapse are often as florid as in perfect health.

In ordinary asphyxia there is capillary engorgement of the lungs, they contain an excess of blood, and are excessively heavy. In cholera collapse the lungs are comparatively anæmic and reduced in weight. In ordinary asphyxia there is capillary stasis in the lungs. In cholera collapse there is an arrest of a large portion of the circulating blood before it reaches the pulmonary capillaries.

In ordinary asphyxia all the abdominal viscera are gorged with dark blood. In cholera collapse these organs, more especially the spleen and the kidneys, are lighter and contain less blood than usual, in proportion to the scantiness of the arterial current which has flowed through them during collapse. But there is congestion of the mucous membrane voL. $\mathbf{L}$. 
of the stomach and intestines, owing to the afflux of blood which attends the elimination of the poison.

Before I suggest an explanation of the arrested circulation in the collapse of cholera, let me briefly point out some of the obvious and necessary results of that remarkable condition.

The stream of blood through the lungs is the channel by which oxygen is introduced into the system. And cateris paribus, the absorption of oxygen into the blood, bears a direct relation to the volume of blood which circulates through the pulmonary capillaries. ${ }^{1}$ Exercise quickens the circulation and the respiration at the same time. The two functions are most intimately correlated. On the other hand, if the current of blood through the lungs be reduced to one quarter or one sixth of its normal amount, as it probably is during the collapse stage of cholera, the respiration becomes feeble and shallow, and the amount of oxygen absorbed is reduced in the same ratio. As a result of this there is defective oxidation of the blood and of the tissues, diminished temperature of the body, diminished exhalation of carbonic acid, and a diminished formation' of biliary and urinary constituents. So that, with an almost complete suppression of bile and urine, there is little or no evidence of their accumulation in the blood during the stage of collapse. Suppression of bile and urine is, as a rule, exactly coincident with collapse. Before collapse the stools are pale, in consequence of their abundant dilution with water; and the urine is scanty because its liquid parts are drained off by the bowel, just as in rheumatic fever they are drained off by the skin. The secretion of bile and urine returns with the returning freedom of the circulation. When suppression of these secretions continues after reaction, this is due to defective action of the excretory glands ; and the constituents of bile and urine then accumulate in the blood.

I refer to acknowledged facts when I say that carbonic

1 There was an element of truth in the doctrine of the ancient anatomists, that the arteries convey "vital air or spirits" from the lungs throughout the body. 
acid, bile, and urine, are joint products of oxidation. There is a constant and most intimate bond between the lung, the liver, and the kidneys. They are physiologically correlated in a very striking manner; and their active work begins simultaneously at the moment of birth. During intra-uterine life, the lungs are entirely inactive : no air is admitted into their cells, and the blood from the pulmonary artery passes directly through the ductus arteriosus into the aorta. The kidneys and the liver are nearly as inactive as the lungs. The bladder, it is true, usually contains some urine; and the meconium which is contained in the intestines is a modified form of bile: but the amount of these secretions formed during intra-uterine life is infinitely small in comparison with the abundant excretion which begins immediately after birth, when, with the establishment of the function of respiration, there is an evolution of carbonic acid, and a continuous formation of the two correlative secretions-the two joint products of oxidation-bile and urine. During the collapse of cholera there is a near approach to that inactive state of the lungs, the liver and the kidneys, which is the natural condition of these organs in the fœtus, and as the primary cause of their joint activity at the moment of birth, is the establishment of the process of respiration; so the essential cause of their conjointly diminished activity during cholera collapse is the partial arrest of blood in the lungs, and the consequent impairment of the function of respiration.

This explanation of the suppression of bile and urine receives confirmation from the fact, which has been noticed by many observers, that if a nursing mother falls into collapse, the secretion of milk, which is not a product of oxidation, continues apparently unchecked, and the breasts become painfully distended. In cases of extreme collapse the secretion of milk may be diminished or suspended in consequence of the extreme scantiness of the blood supply. In like manner, and for the same reason, the secretions from the alimentary canal may be arrested or nearly so. For the continuance of any secretion there are two essential 
conditions: 1. A due supply of moving blood to the secreting tissue; 2, The presence in the blood of the constituent elements of the secretion, or the materials out of which the secretion may be formed.

Another result of defective oxidation during collapse is a diminution in the amount of fibrin in the blood and an excess of colouring matter. On the other hand excessive oxidation increases the fibrin and lessens the amount of red corpuscles.

The diminished absorption during collapse is obviously a result of the defective circulation. Cateris paribus absorption is active in proportion to the rapidity of the bloodcurrent. During collapse the absorption of gases by the lungs, and of liquids by other tissues and organs, is lessened in proportion to the smallness and the feebleness of the bloodstream. Without doubt, too, the absorption of materials from the intestinal canal is impeded by the outward current from the blood-vessels into the bowel. Another circumstance which influences the rate of absorption is the degree of emptiness of the blood-vessels; when, during the stage of reaction the circulation again becomes free, the comparatively empty condition of the vessels renders absorption very active, and there is a probability that morbid secretions which have been retained within the bowel may then be reabsorbed and become a source of danger.

There is no disease which serves so well as cholera to illustrate the close relationship which exists between the functions of circulation, respiration, secretion, and absorption.

Here I would suggest that during collapse the contractile power of the heart is probably impaired, not by the direct action of a poison, but in consequence of the defective supply of blood through the coronary arteries.

Reverting now to the arrest of the pulmonary circulation, can we explain it? It has been supposed by some that the blood has become so thickened by the loss of its water that it cannot pass through the minute vessels of the lungs. The objections to this theory are, that the onset of collapse is 
often too sudden to be thus explained; that the circulation is restored and the symptoms of collapse pass away, while the discharge of fluid continues; that the symptoms of obstructed circulation bear no direct relation to the loss of fluid, often rather an inverse relation exists between them; that the anæmic condition of the lungs in collapse proves that the obstruction does not begin in the capillaries, where it obviously must first occur if the theory in question were true. Lastly, that the blood is not found remarkably thickened; on the contrary, when examined soon after death it is usually very liquid, and it escapes with great rapidity when a distended vein is punctured in opening the chest.

A comparison of the physiological symptoms of collapse with the anatomical condition of the lungs after death clearly indicates that the arrest of the circulation begins in the minute arteries. We know that the minute arteries have muscular walls; that when examined under the microscope in the transparent parts of living animals (the bat's wing or the frog's foot) they may be seen, when stimulated by cold or electricity, or mechanical irritants, to contract to such a degree as to obliterate their canals, and so for a time to greatly diminish or entirely arrest the flow of blood through the parts which they supply. The experiments of Hales, ${ }^{1}$ performed a century ago, prove that the arteries of animals immediately after death, so long as the vital contractility remains, have the power to resist the passage of such fluids as, by their low temperature or their irritating qualities, excite the contractility of the minute vessels.

Mr. James Blake has shown by numerous careful experiments $^{2}$ that certain salts injected into the veins of a dog immediately arrest the circulation through the lungs. The salts of soda have this effect. The animal dies in less than a minute, and after death the left side of the heart is found empty, while the right cavities are over-distended. When air is blown into the veins of an animal, or when, in operations about the root of the neck or axilla, it is

' 'Statical Essays,' 1769.

2 'Edinburgh Medical and Surgical Journal,' vols. lii, liv, lvi. 
accidentally sucked into the veins of the human subject, it causes great obstruction to the pulmonary circulation, and often sudden death. The left side of the heart, as in Blake's experiments, is found nearly empty, while the right cavities and the pulmonary artery are distended with frothy blood. Mr. Erichsen found, by experiments on a dog recently killed, that blood mixed with air required nearly twice the pressure to drive it through the pulmonary vessels that would suffice to drive unmixed blood through the lungs.

The results of these experiments and accidents afford illustrations of the influence which the contractility of the minute arteries exerts upon the stream of blood. The minute arteries alone have this contractile power, and the effect of their contraction is, not to propel the blood onwards, but to narrow the stream or to arrest it entirely, as a stop-cock lessens or stops the flow of water. Dr. Marey's most interesting book ('Physiologie Médicale de la Circulation du Sang') contains abundant proofs and illustrations of the controlling influence which the contractility of the small vessels exerts upon the blood-current. The phenomena of the pulse in health and the variations of the pulse in disease are utterly unintelligible without constant reference to the contraction of the minute arteries. The only known natural agency which has the power to stop abruptly liquid blood in its passage through the vessels is the contraction of the small arteries. I say liquid blood, for it is obvious that vessels of large size may be mechanically obstructed by emboli, and when a clot suddenly obstructs the pulmonary artery the superficial symptoms-shrinking of the features, a mixture of blueness with pallor, coldness and pulselessnessare essentially the same as those which occur in cholera collapse. Although embolism is sometimes a consequence of the obstructed pulmonary circulation in cholera, it certainly is not the cause. And I repeat that the only known agency which will explain the arrest of blood is arterial spasm, a spasm which may come and go as gradually or as suddenly. as bronchial spasm, and which, like that, may be of longer or shorter duration, dependent upon the persistence of the 
exciting cause. It is, of course, as impossible to demonstrate the existence of spasm of the pulmonary arteries as to demonstrate that of the bronchial tubes. In both instances the theory of spasm is an inference from a number of wellascertained facts, and the only way in which either theory can be refuted is to show that it is inconsistent with unquestionable facts. Those who, in such a case, will be convinced by nothing short of demonstrative evidence must remain sceptical until they become endowed with supernatural powers of vision.

It is interesting to compare the phenomena of spasmodic asthma with those of cholera collapse. In both diseases spasm is the main cause of the morbid phenomena. In the one the spasm is bronchial, in the other it is arterial. In both diseases there are defective respiration and impeded circulation ; in one the defective respiration is primary, and the impeded circulation is a secondary result of that ; in the other the phenomena occur in the reverse order. In both diseases there is evidence of the intimate relationship which exists between the function of respiration and the circulation through the lungs. In both diseases there is feebleness of voice, and in both this is mainly due to the small volume and force of the respired current of air; "the vocal pipe, feebly blown through, refuses to speak."

The theory of arterial spasm receives striking confirmation from the effect of hot injections into the veins during collapse. It has been very generally supposed that the great relief which in most cases immediately follows this operation is due to the mere addition of liquid to the blood. All the facts and arguments which are opposed to the theory that the loss of water is the main cause of collapse render it improbable that the mere addition of water to the blood would remove that condition. Some have supposed that the salts, dissolved in the water, might exert a peculiar vitalising influence on the blood. But it has been proved that there is no deficiency of salts in the blood of cholera patients, and it has been found repeatedly that hot water alone has the same effect as the injection of a saline solution. 
Dr. Mackintosh, who in the year 1832 injected 156 patients, soon ascertained that the hotter the liquid the more immediate and striking was the effect. The probable cause of the arterial spasm is the irritating quality of the cholera blood; and the probable action of the injection into the veins is twofold. The mere addition of water at any temperature would dilute the morbid blood and render it less irritating to the vessels. The injection of hot water would, in addition, have a direct effect in relaxing the arterial spasm. It has been observed that if, by any external application of heat, the patient can be thoroughly warmed, the pulse and the other symptoms of collapse are simultaneously improved.

As the effect of hot injections is to improve the pulse by relaxing arterial spasm, so the effect of alcoholic stimulants during the stage of collapse is to increase the obstruction in the lungs, and thus to lessen the volume and force of the pulse.

But, it has been asked, if the cholera poison excites spasm of the pulmonary arteries, why has it not a similar influence upon the systemic arteries? I cannot answer this question except by pointing to facts which show that the systemic and the pulmonary arteries have different vital endowments, so that blood of a certain quality may be readily transmitted. by one set of vessels, yet be violently opposed by the other. It is the function of the pulmonary arteries to convey black blood, and they do it readily; but when from any cause black blood enters the systemic arteries, its passage through the extreme vessels is resisted, and the whole arterial system becomes in consequence distended. Again, Blake ascertained that the salts of ammonia and of potash, when injected into the veins of a dog, pass readily through the pulmonary vessels, but are resisted in their passage through the systemic vessels.

It has been said that, admitting the existence of arterial spasm, this is a result of the action of the cholera poison upon the spinal cord or upon the sympathetic ganglia. Obviously the vaso-motor nerves must have an influence upon the vascular spasm. But that the immediate cause of 
the arterial spasm in the collapse of cholera is the direct action of the poisoned blood upon the inner surface of the vessels, appears to be proved by the influence of the hot injections in removing the spasm. These injections would obviously have a direct local action upon the vessels, but it is not probable that they could exert an immediate influence upon the spinal cord or the sympathetic ganglia. The contraction of the minute arteries appears to be the result of a reflex action of the nerves upon the muscular tissue, and it is analogous to the spasm of the glottis which is excited by a drop of water or a crumb of bread in the laryux. It is probable that the same morbid poison which excites the arterial spasm is the cause of the painful cramps of the voluntary muscles, and of those remarkable muscular twitchings which often occur after death.

In connection with this subject it is interesting to remark that Blake observed that when a dog has been killed by injecting a solution of a salt of either baryta, strontia, or lead, post-mortem twitchings occur, which are evidently very similar to those observed in cholera, though Blake himself makes no allusion to cholera. With reference to the question whether these muscular contractions are due to the action of the poison upon the nervous system or directly upon the muscles, Blake mentions one fact which, as he says, is in favour of the latter mode of action. "When these substances have been injected into the arteries the contractions are generally most marked in the limb through the artery of which the injection has been introduced."1

Bernard has proved that the influence of the wourara poison may be cut off from the posterior limbs of a frog by ligaturing the abdominal aorta and all the soft parts except the lumbar nerves before the poison is inserted. The effect of the poison is to destroy the power of the motor nerves to transmit either the electrical or the voluntary nervous stimulus, while the nerves of sensation and the contractile power of the muscles remain intact. A frog prepared in the manner described, and then poisoned by wourara, manifests

1 'Edinburgh Medical and Surgical Journal,' vol. lvi, p. 116. 
pain when his anterior limbs are pinched, by struggling with his hind limbs, while his fore limbs, paralysed by the poison, remain quite motionless. The poison, as Bernard explains, is conveyed by the blood, and acts first upon the extremities of the motor nerves, its action spreading from the periphery towards the spinal cord but not in the opposite direction. $^{1}$

The pathological significance of the discharges from the alimentary canal.-It seems reasonable to suppose that the choleraic discharges from the alimentary canal are analogous to the profuse sweats of rheumatic fever and to the eruption on the skin in cases of smallpox and other exanthemata. They are outward manifestations of a morbid change in the blood; and inasmuch as they are of constant occurrence during the process of recovery, we must infer that they are a necessary part of that process. It is probable that the cholera poison, having entered the blood, undergoes a rapid process of self-multiplication, and spoils certain of the blood-constituents, which are then ejected through the mucous membrane of the alimentary canal. If the action of the poison be moderate in degree the disease does not pass beyond the stage of vomiting and purging, and the severity of the attack is measured by the amount of discharge from the alimentary canal. If, however, the poison be more virulent, or the patient more susceptible of its influence, the blood speedily becomes so changed that the pulmonary vessels resist its passage, and the state of collapse occurs. The impeded circulation, in proportion to its degree, interferes with the process of elimi. nation, and thus there may be an inverse ratio between the symptoms of collapse and the gastro-intestinal symptoms. The state of collapse, therefore, is one of great peril, and while it continues the blood is being spoiled, partly by the zymotic action of the morbid poison, partly by the suspension or rather the imperfection of the respiratory changes.

When after the elimination of the poison and its products the circulation again becomes free, the blood

1 'Leȩons sur les Propriétés des 'Tissus Vivants,' 1866, p. 177. 
contains an excess of unoxidized materials which now rapidly combine with oxygen. Bile, urine, and carbonic acid are abundantly formed, sometimes more rapidly than they can be excreted; they consequently accumulate in the blood; the lungs, the liver, and the kidneys-one or allbecome gorged, and there is a state of consecutive fever scarcely less perilous than that of collapse.

I have spoken of the discharges from the alimentary canal as being a necessary part of the curative process; but are they not a source of exhaustion? Unquestionably they are, and they may be fatal by their abundance. This I suspect is not infrequent when patients living in an infected district imbibe or inhale daily fresh doses of the poison, so that the frequent discharges increase exhaustion, but leave the system still infected by renewed doses of the morbific agent.

The only way in which a diarrhœa thus excited and perpetuated can be surely and safely stopped is by the discovery and avoidance of the exciting cause in the poisoned air or water. The action of repressive drugs while the cause continues in operation is either nil or mischievous.

The principle of treatment.-Patients have recovered from cholera in all its stages under the most varied and opposite methods of treatment, and without any treatment at all. It is therefore manifest that there is a natural process of cure. In speaking of the cure it is convenient to divide the process into three stages-1st, blood zymosis ; 2nd, vascular excretion of morbid products from the blood into the bowel ; 3rd, gastro-intestinal evacuation by vomiting and purging.

Is there any specific cure for the disease-any means by which the blood changes can be prevented or arrested ? I know of none.

Can we check the process of vascular excretion? Probably opium may have this effect, and there is reason to believe that in proportion as it does this it increases the risk of fatal collapse. Should we endeavour to increase the discharges from the blood? It is doubtful whether any 
drug would have the effect, even if it were desirable, which it is not. So long as the circulation is free the amount of vascular excretion will be an exact measure of the dose or the virulence of the poison to be eliminated, just as in diabetes the measure of urine secreted is an index of the amount of sugar to be discharged. When the process of vascular excretion is checked by the arrest of the circulation, the only way in which we can render any effectual aid is by setting free the circulation. This may sometimes be accomplished by hot injections into the veins and by venesection. I have before explained the action of the venous injections. Venesection probably acts by relieving over-distension of the right cavities of the heart, and perhaps it may tend to lessen spasm. In the third stage of the natural process of curethat of gastro-intestinal evacuation - the object is to prevent the accumulation of the morbid secretions in the alimentary canal, and to ensure their speedy expulsion. Further, to avoid narcotic and repressive means until the process of expulsion has been well-nigh completed.

I purposely avoid entering into details. If we can agree upon a principle of treatment, we may greatly assist each other in working out the details.

Many practitioners believe that by opiates and astringents in the diarrhœa stage they prevent the disease passing into the stage of collapse. Their belief would be less open to question if every diarrhœa thus treated were an early stage of cholera, which tended inevitably to pass into collapse when not arrested by remedies. But is this so? I believe not. With reference, however, to this question, rather than give my own experience, of which I have said enough elsewhere, I will refer to that report on cholera in Malta to which I have before alluded. The authors state that during the epidemic three forms of diarrhœea were met with1. The ordinary summer diarrhœa, which was easily checked and appeared to have no relation to cholera. The second form was characterised by painless watery purging, often associated with vomiting. It was found in every degree of intensity, and when severe was classed under choleraic diar- 
rhœa. Although intractable, it evinced no tendency to pass beyond a certain point or to assume a more malignant form. The third form of diarrhœa was an intensification of the second kind, and so completely intractable that in sixty-one cases, when every possible attempt was made to check it, in none did it succeed, but it was invariably followed by full development of cholera. In the words of the authors of this most instructive report, "The second variety showed no tendency to pass beyond a certain point if not stopped. Its severe form, the third variety, was clearly an early stage of cholera; and it may be fairly questioned whether a single case was prevented developing into cholera by treatment directed towards the suppression of the intestinal flux." Upon this statement I would remark that the repressive means which were employed in these cases were probably worse than useless. If the account which I have given of the pathology of cholera be true or nearly so, then it is certain that the indiscriminate employment of opiates and astringents in the diarrhœa stage must favour the tendency to collapse by impeding the exit of the poison from the blood and from the alimentary canal. I have seen the worst symptoms of collapse follow so directly upon the arrest of a diarrhœa by opinm as to render it nearly certain that the phenomena stood to each other in the relation of cause and effect.

Of cholera it may be said, as of many other acute diseases, that for the cure of most cases that are curable by any means the vis medicatrix natura will suffice. Yet there are few cases in which we cannot render some assistance, and not a few in which by a discreet co-operation with nature we may turn the scale and save a life which without our aid would have been lost.

Lastly, the one great lesson which cholera is designed to teach us is a lesson of cleanliness, especially in the matter of our water supply. There is no disease which so forcibly impresses upon us the necessity of a cathartic method of treatment in the widest sense of that term. If we convert our rivers into sewers they must cease to be the source 
of our water supply. The philosophic Hamlet in imagination traces the dust of Alexander until he finds it stopping a bung-hole. At the present day it requires little exercise of the fancy to suppose it possible that a resident in London, on a visit to Oxford, may there deposit certain organic matters which may be restored to him in his drinking water after his return to London. It is conceivable that the same identical particle of urea might pass a second time through the kidneys of a man who drinks water from a common sewer. Unless we put a stop to this strange circulation of loathsome organic impurities our blood-vessels will refuse to transmit the intolerable stuff that we put into them. To swallow sewage, and then by repressive drugs to prevent its expulsion from the body is the very climax of irrational and destructive treatment. The drinking of sewage, however, is probably not in itself a sufficient cause of cholera; but during an epidemic season there appears to be superadded to the sewage a something which renders it a specific poison; or it may be that the effect of impure water is to render the body susceptible of the action of an atmospheric poison. It may be that the cholera poison is the product of two noxious agents, one entering the body with the air through the lungs, the other with the water through the alimentary canal.

Postscript.-It has been objected to the theory of arterial spasm as the cause of cholera collapse, that, if the theory were true, opium or chloroform would remove the symptoms of collapse by relaxing the spasm. This objection is based upon the assumption that these drugs have the power to relax arterial spasm; an assumption entirely unsupported by evidence. We know that there are forms of spasm over which the drugs in question appear to have no influence. Opium will not relax the spasms of tetanus; and chloroform, while it annihilates the pains of labour, does not arrest the uterine contractions. The assumption that either opium or chloroform has the power to overcome spasm of the arteries is quite gratuitous, and therefore the objection referred to has no weight. 
Pagei2' *

\section{DiagramfTable 4/showing the}

Mortality Per Cent, from Chotera, at different ages, and under defferent modes of treatment.

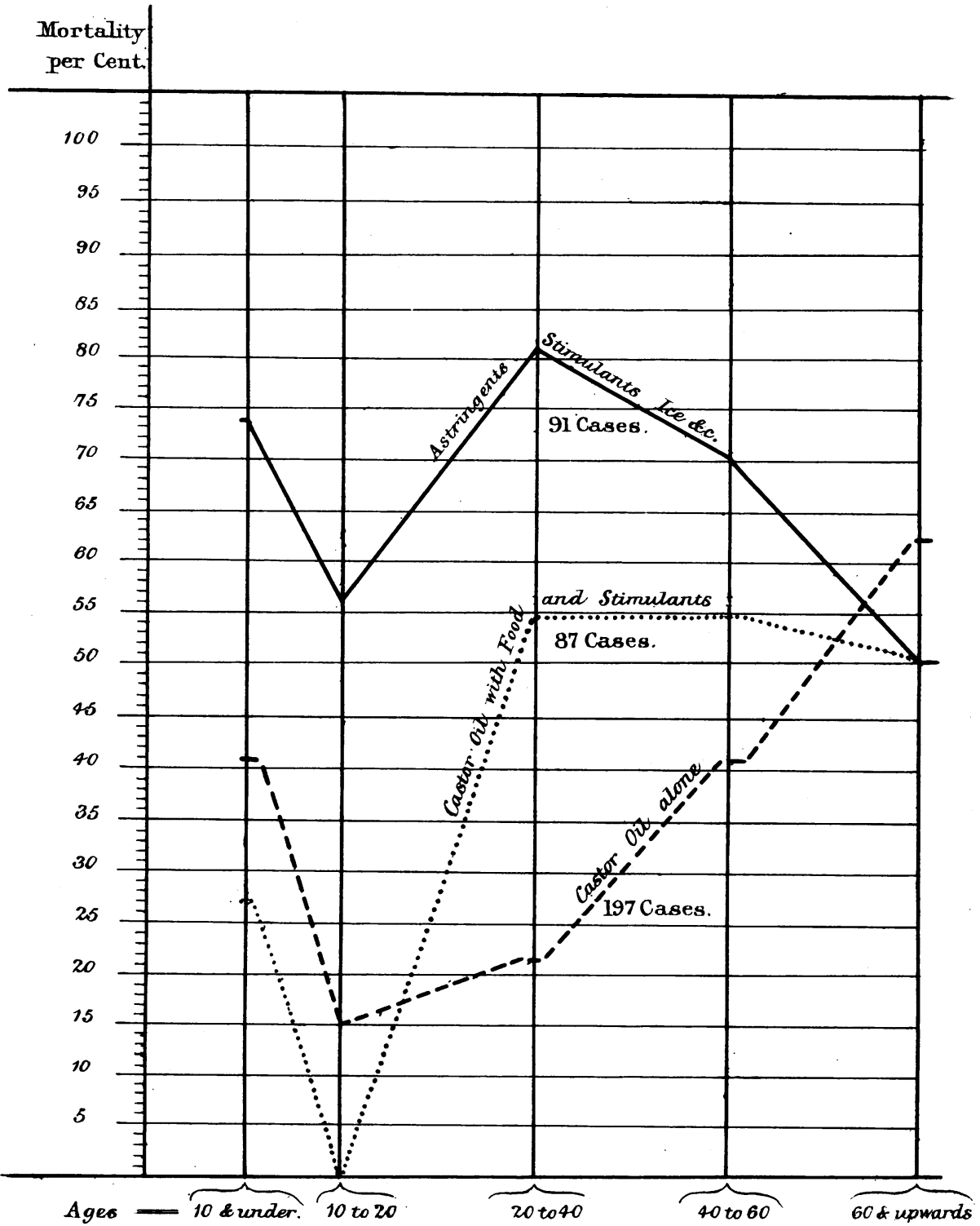

